\title{
Profiles of tumor-infiltrating immune cells in renal cell carcinoma and their clinical implications
}

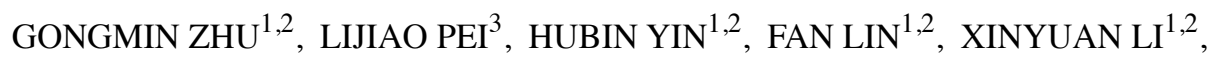 \\ XIN ZHU ${ }^{1,2}$, WEIYANG HE ${ }^{1}$ and XIN GOU ${ }^{1}$ \\ ${ }^{1}$ Department of Urology, The First Affiliated Hospital of Chongqing Medical University; \\ ${ }^{2}$ Chongqing Key Laboratory of Molecular Oncology and Epigenetics, Chongqing 400016; ${ }^{3}$ The State Key \\ Laboratory of Biotherapy, West China Hospital, Sichuan University, Chengdu, Sichuan 610041, P.R. China
}

Received April 26, 2019; Accepted August 30, 2019

\section{DOI: $10.3892 / 01.2019 .10896$}

\begin{abstract}
Tumor-infiltrating immune cells (TIICs) are crucial for the clinical outcome of renal cell carcinoma (RCC), as they regulate cancer progression. TIICs have therefore the potential to become novel targets of immunotherapies. The present study used CIBERSORT analytical tool, which is a deconvolution algorithm, to comprehensively analyze the composition of immune cells in RCC and normal tissues from The Cancer Genome Atlas (TCGA) cohort, and to determine the prognostic value of TIICs in RCC. A landscape of infiltrating immune cells was determined as containing 13 subpopulations of immune cells, with significant differences between normal and tumor tissues. Subsequently, Kaplan-Meier analysis and log-rank test were used to estimate the prognostic value of TIICs in RCC. The results demonstrated that a higher proportion of regulatory $\mathrm{T}$ cells (Tregs) [hazard ratio $(\mathrm{HR})=1.596$; 95\% confidence interval $(\mathrm{CI}), 1.147-2.222 ; \mathrm{P}=0.006]$ and follicular helper T cells (HR=1.516; 95\% CI, 1.089-2.111; $\mathrm{P}=0.014)$ were associated with poor outcome in patients with RCC. Conversely, resting mast cells ( $\mathrm{HR}=0.678$; 95\% CI, 0.487-0.943; $\mathrm{P}=0.021)$ and monocytes $(\mathrm{HR}=0.701 ; 95 \%$ CI, 0.503-0.977; $\mathrm{P}=0.036$ ) were associated with a favorable prognosis in patients with RCC. Furthermore, the results from multivariate Cox regression analysis indicated that Tregs and monocytes represented independent risk factors for prognosis in patients with RCC. These findings demonstrated that gene profiling deconvolution by CIBERSORT served to determine the composition of immune cells infiltrated in RCC and may provide some crucial information for the development of immunotherapies.
\end{abstract}

Correspondence to: Professor Xin Gou, Department of Urology, The First Affiliated Hospital of Chongqing Medical University, 1 Youyi Road, Yuzhong, Chongqing 400016, P.R. China E-mail: gouxincq@163.com

Key words: tumor-infiltrating immune cells, renal cell carcinoma, prognosis, The Cancer Genome Atlas, bioinformatics analysis

\section{Introduction}

Renal cell carcinoma ( $R C C$ ) is the most common type of kidney carcinoma and the sixth and tenth most frequent type of cancer diagnosed in men and women, respectively (1). The mortality rate of RCC reaches 140,000 cases per year worldwide, and RCC ranks as the 13th leading cause of cancer-associated mortality (2). At present, surgical resection remains the preferred curative therapy for RCC; however, since $\sim 1 / 3$ of patients with RCC are diagnosed with advanced or metastatic $\mathrm{RCC}$, their prognosis remains unsatisfactory following curative operation $(3,4)$. In the past few decades, emerging evidence demonstrated that the malignant phenotypes of tumors are determined by the intrinsic activities of cancer cells and by the complex interactions of various cell types in the tumor microenvironment, in particular tumor-infiltrating immune cells (TIICs) $(5,6)$. Previous clinical and genomic studies reported that RCC is an immunogenic tumor $(7,8)$. Furthermore, the cases of spontaneous and significant remission of RCC are also considered to be mediated by the immune response $(9,10)$. In addition, immunotherapies have been used in clinical trials of RCC alone or in combination with targeted therapies, and have been demonstrated to prolong patients' survival (11-13). It is therefore crucial to determine the underlying mechanisms of tumor microenvironment infiltration with immune cells in order to improve the immunotherapeutic approaches to treat RCC. However, whether immune cells represent a critical component of tumor microenvironment remains poorly understood.

TIICs have been reported to function as tumor promoters or suppressors in various types of cancer and to have great prognostic value. For instance, tumor-associated macrophage infiltration has a negative impact on prognosis in patients with breast and bladder carcinomas, whereas $\mathrm{CD} 8^{+}$cytotoxic $\mathrm{T}$ cells and CD45RO+ memory $\mathrm{T}$ cells, the shortest CD45 isoform which lacks all three of $\mathrm{A}, \mathrm{B}$, and $\mathrm{C}$ regions, are associated with longer overall survival (OS) and disease-free survival (DFS) $(14,15)$. Recent studies demonstrated that RCC is also infiltrated by heterogeneous TIICs, including macrophages, $\mathrm{T}$ cells and $\mathrm{B}$ cells, and that their location, density and type in RCC are associated with prognosis $(16,17)$. However, TIICs have largely been characterized using tissue-based approaches, 
including immunohistochemistry (IHC) and flow cytometry, which may be limited by various factors, including the amount of tissue required, and that the number of immune cell types may not be evaluated simultaneously. These aforementioned approaches may therefore not lead to comprehensive and convincing conclusions.

A novel gene-based approach named CIBERSORT has been described; this computation method applies deconvolution of a reference gene expression matrix and support vector regression to estimate the fractions of various cell types, including TIICs, in complex tissues $(18,19)$. The diversity and landscape of TIICs can therefore be determined by this approach. The present study used CIBERSORT analytical tool to deconvolute gene expression data and determine 22 immune cell types in RCC tissues, and further investigated their clinical significance.

\section{Materials and methods}

Data acquisition from the cancer genome atlas (TCGA) cohort. Gene expression profiles of RCC $(n=895)$ and normal samples $(n=128)$, and clinical characteristics from the corresponding patients were downloaded from the TCGA data portal in March 2019 (20). RNA sequencing data were normalized with the mean-variance modeling at the observational level (voom) method (21), which converted count data to values more similar to those resulting from microarrays. For clinical data, only patients with RCC and complete information $(n=421)$ were included. Patients with RCC who had missing data for age, gender, Fuhrman grade, Tumor-Node-Metastasis (TNM) classification, survival time or status were excluded.

Evaluation of TIICs. CIBERSORT analytical tool is a deconvolution algorithm that applies a set of barcode gene expression values that corresponds to a 'signature matrix' of 547 genes to precisely determine the immune cell composition in data from bulk tumor samples of mixed cell types (18). To quantify the proportion of 22 immune cell types in RCC tissues, normalized gene expression datasets were prepared, and data were uploaded to the CIBERSORT web portal (http://cibersort. stanford.edu/), with the algorithm run and the number of permutations being set to 1,000. A total of 22 types of TIICs together with CIBERSORT metrics, including CIBERSORT P-value, Pearson's correlation coefficient and root mean square error, were quantified for each sample. The statistical significance of the deconvolution consequences of all cell subsets was reflected by the CIBERSORT P-value, which was used to filter out deconvolution with a less significant fitting accuracy. To meet the requirement of a CIBERSORT $\mathrm{P} \leq 0.05$, normal samples $(n=17)$ and RCC samples $(n=617)$ were selected.

IHC. Specimens from 20 patients diagnosed with RCC at the First Affiliated Hospital of Chongqing Medical University (Chongqing, China) between February 2017 and October 2018 and who received tumor resection surgery were used for IHC. All specimens were fixed with $4 \%$ formalin at room temperature for $24 \mathrm{~h}$, embedded with paraffin and sliced into $4-\mu \mathrm{m}$ sections and stored at $-20^{\circ} \mathrm{C}$. For IHC, sections were deparaffinized with $100 \%$ xylene for $30 \mathrm{~min}$ at room temperature and rehydrated with a decreasing gradient of ethanol for $30 \mathrm{~min}$ at room temperature $(100 \%$ for $7 \mathrm{~min}$, $95 \%$ for $5 \mathrm{~min}, 85 \%$ for $5 \mathrm{~min}$ and $70 \%$ for $5 \mathrm{~min}$ ). Sections were immersed in a boiling sodium citrate-hydrochloric acid buffer solution and heated in a microwave at $96-98^{\circ} \mathrm{C}$ for the antigen retrieval for $25 \mathrm{~min}$. After cooling at room temperature, $3 \%$ hydrogen peroxide was used to block endogenous peroxidase at room temperature for $10 \mathrm{~min}$. Subsequently, sections were washed three times with TBS and blocked with normal goat serum at room temperature for $15 \mathrm{~min}$. Sections were incubated with a monoclonal antibody against forkhead box P3 (FOXP3; 1:50; cat. no. 98377; Cell Signaling Technology, Inc.) overnight at $4^{\circ} \mathrm{C}$. Sections were then washed three times with TBS and incubated with a goat anti-rabbit immunoglobulin $\mathrm{G}$ secondary antibody at $37^{\circ} \mathrm{C}$ for $30 \mathrm{~min}$. Sections were washed three times with TBS and incubated with streptavidin-biotin-conjugated horseradish peroxidase substrate at $37^{\circ} \mathrm{C}$ for another $30 \mathrm{~min}$. The $3 \%$ hydrogen peroxide, normal goat serum, secondary antibody, and streptavidin-biotin-conjugated horseradish peroxidase substrate were included in an IHC kit (cat. no. SP9001; ZSGB-BIO). Signal was visualized with diaminobenzidine (ZSGB-BIO), and sections were counterstained with hematoxylin at room temperature for approximately $10 \mathrm{sec}$. Sections were analyzed as previously described (19). Briefly, Treg density was determined according to the percentage of positive cells normalized to the total number of cells under a light microscope, at x400 magnification.

Statistical analysis. Statistical analyses were performed with SPSS 22.0 software (IBM Corp.), GraphPad Prism 5.0 software (GraphPad Software, Inc.) and R software (version 3.5.2; $\mathrm{R}$ Foundation). Kaplan-Meier analysis was used to determine survival curves that reflect the association between immune cells and corresponding clinical follow-ups, which were evaluated by the log-rank test. The median value of the proportion of each cell type was used for Kaplan-Meier analysis and modeled as continuous variable to obtain interpretable hazard ratios (HRs). Kruskal-Wallis test was used to analyze the association between TIICs and clinical information (Fuhrman grade and TNM classification). Univariate and multivariate Cox regression analyses were used to estimate the prognostic significance of the inferred TIICs. Two tailed Student's t-test was used to examine the statistical relevance between two groups. $\mathrm{P}<0.05$ was considered to indicate a statistically significant difference.

\section{Results}

Profile of immune infiltration in RCC and normal samples. According to the CIBERSORT algorithm, the 22 immune cell types infiltrated in RCC samples were investigated and their proportions were visualized. The results revealed the profile of immune infiltration in RCC samples. The bar plot demonstrated the results from 17 normal samples and 617 RCC samples. The proportions of immune cells were variable in each sample (Fig. 1A). In addition, unsupervised hierarchical clustering was used to identify the subpopulations of immune cells. The normal and tumor samples were divided into two discrete groups, and the proportions of TIICs varied between these groups (Fig. 1B). These results suggested that the variation of TIICs proportions may be an essential 


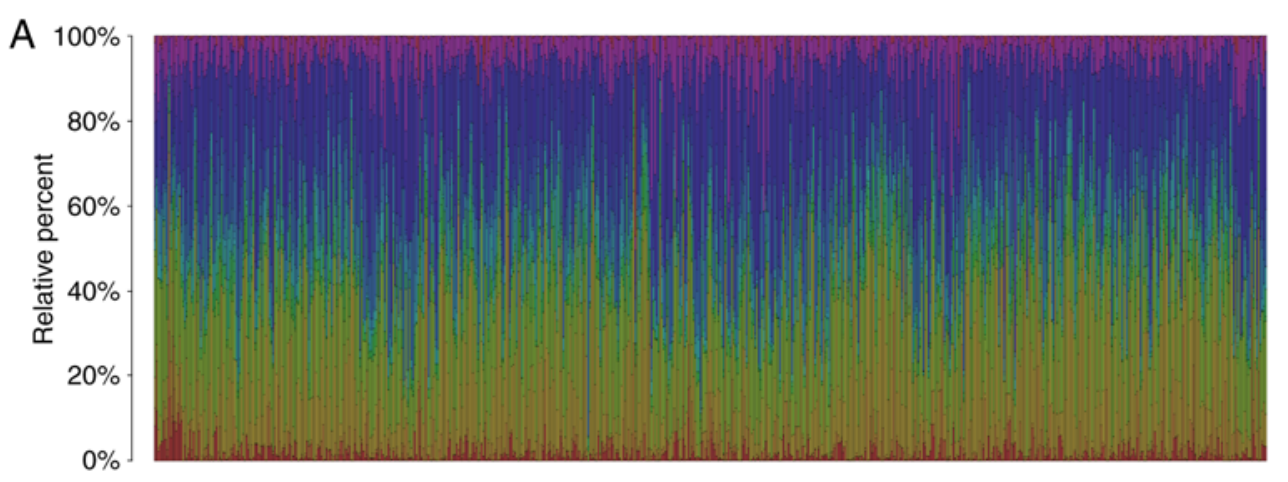

- B cells naive

B cells memory

" T cells CD8

- T cells CD4 naive

- T cells CD4 memory resting

" T cells CD4 memory activa

- T cells follicular helper

- T cells gamma delta

" NK cells resting

- Monocytes

- Macrophages MO

- Macrophages M1

- Mendritic cells resting

Mast cells resting

- Mast cells activated

" Eosinophils
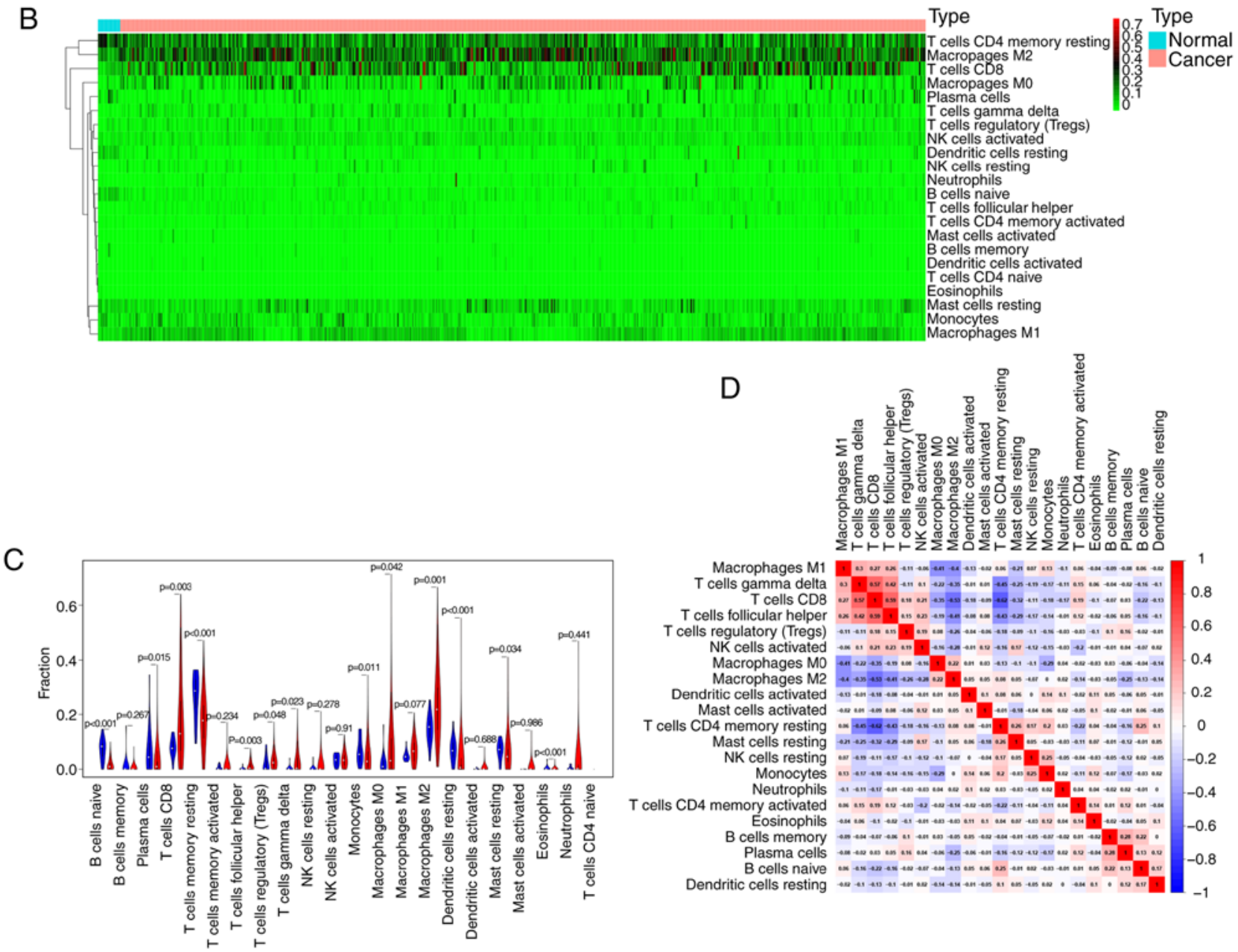

Figure 1. Profile of immune infiltration in RCC and normal samples. (A) Stacked bar chart representing deviations in immune infiltration in each sample. (B) Hierarchical clustering of normal and RCC samples based on 22 immune cell proportions. The horizontal axis represents samples that were divided into two clusters. Red, black and green respectively indicates the high, moderate and low proportion of immune cells, respectively. (C) Difference in proportion of each immune cell in normal and RCC tissues. Blue represents normal samples and red represents RCC samples. $\mathrm{P}<0.05$ represents statistical significance. (D) Correlation matrix of immune cell proportions. The red color represents positive correlation and the blue color represents negative correlation. RCC, renal cell carcinoma.

characteristic that could describe the individual differences. As presented in Fig. 1C, the differences in each immune cell between normal and RCC samples were further analyzed. The results demonstrated that 13 immune cells were significantly different between the two sample types. In particular, CD8 $\mathrm{T}$ cells, follicular helper T cells, Tregs, $\gamma \delta \mathrm{T}$ cells, and M0 and M2 macrophages were more present in RCC samples than in normal samples, whereas B cells naïve, plasma cells, CD4 memory resting $\mathrm{T}$ cells, monocytes, resting dendritic cells, resting mast cells and the eosinophil fraction were lower in
RCC samples compared with those in normal samples. These TIICs may therefore be involved in the development of RCC and may be associated with the prognosis of patients with RCC. Furthermore, the results from the correlation matrix demonstrated that follicular helper $\mathrm{T}$ cells had the strongest positive correlation with CD8 T cells, whereas CD4 memory resting $\mathrm{T}$ cells had the strongest negative correlation with CD8 T cells, and that the proportions of other TIICs subpopulations presented a weak to moderate correlation (Fig. 1D). Taken together, these findings suggested that the deviations 

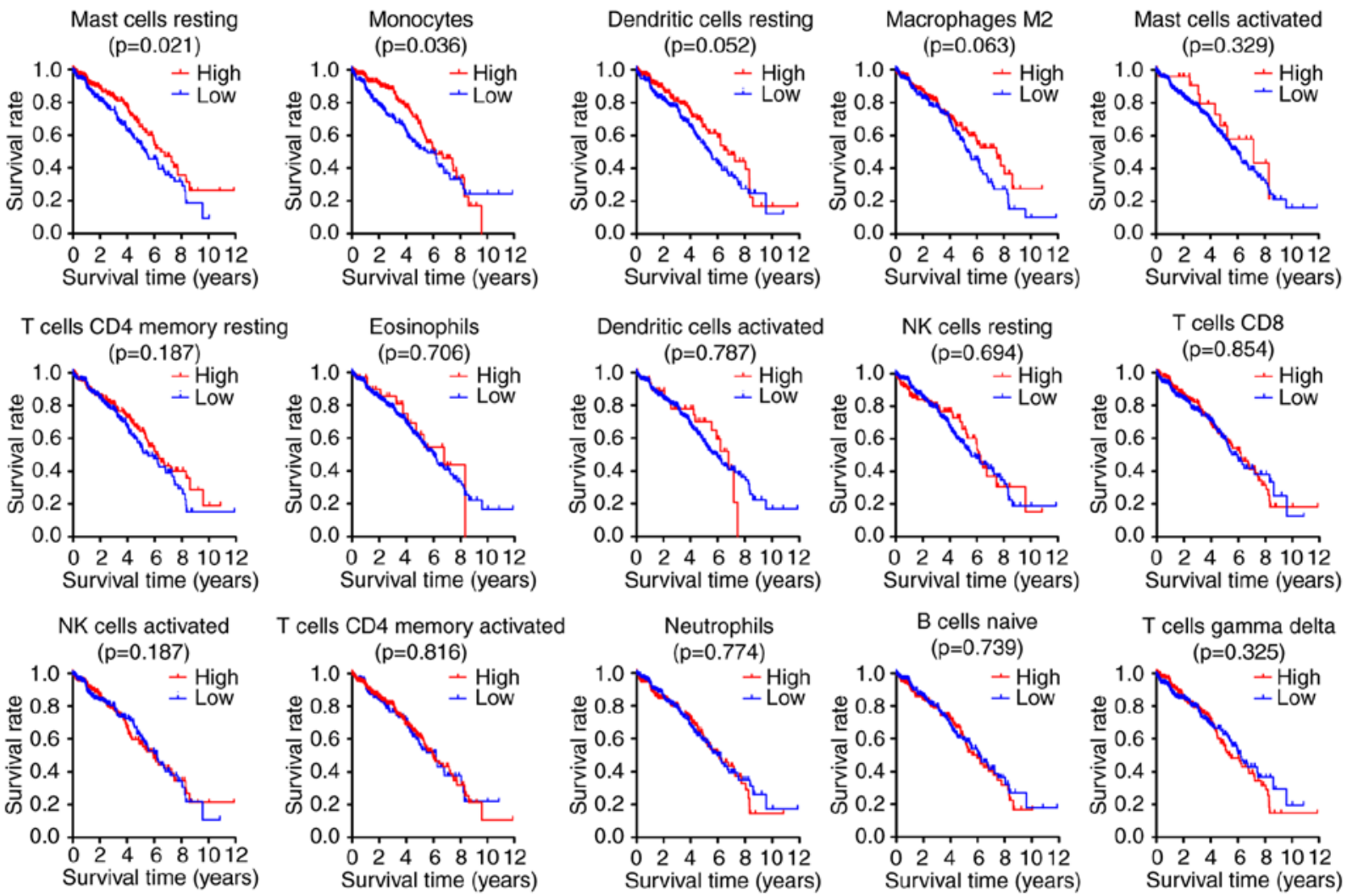

T cells CD4 memory activated
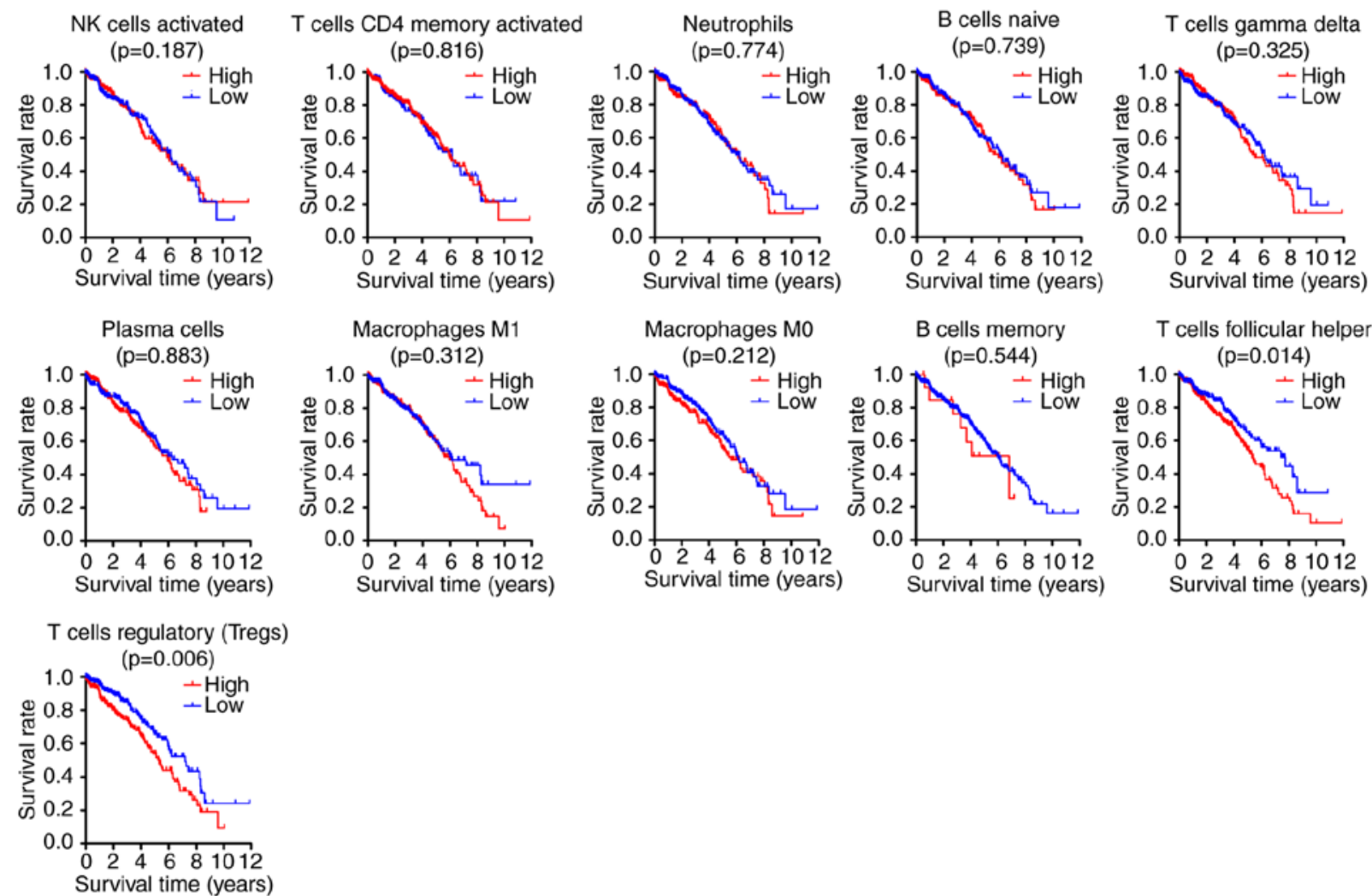

Figure 2. Kaplan-Meier survival curves for tumor-infiltrating immune cells in renal cell carcinoma samples (n=421).

of the RCC immuno-landscape from normal tissue may have significant clinical implications.

Prognostic value of TIICs in RCC samples. The correlation between prognosis of patients with RCC and TIIC subpopulations was further investigated. Following Kaplan-Meier analysis and log-rank test, the results demonstrated that increased proportion of resting mast cells [HR $=0.678 ; 95 \%$ confidence interval (CI), 0.487-0.943; $\mathrm{P}=0.021]$ and monocytes $(\mathrm{HR}=0.701 ; 95 \% \mathrm{CI}, 0.503-0.977 ; \mathrm{P}=0.036)$ was associated with a favorable outcome, whereas a greater proportion of follicular helper T cells $(\mathrm{HR}=1.516 ; 95 \% \mathrm{CI}, 1.089-2.111 ; \mathrm{P}=0.014)$ and Tregs $(\mathrm{HR}=1.596 ; 95 \% \mathrm{CI}, 1.147-2.222 ; \mathrm{P}=0.006)$ was associated with poor prognosis (Figs. 2 and $3 \mathrm{~A}$ ). In addition, the association between these four immune cell types and patients' clinical information was determined. As presented in Fig. 3B and $\mathrm{C}$, Tregs and follicular helper T cells were positively associated with Fuhrman grade and TNM classification, whereas resting mast cells were negatively associated with Fuhrman grade and TNM classification. Furthermore, monocytes were also negatively associated with Fuhrman grade, but was not associated with TNM classification.

Cox regression model for $O S$. To determine whether inferred TIICs were independent risk factors for the prognosis of patients with RCC, Cox regression analysis was used. Univariate analysis demonstrated that age, grade, TNM classification, $\mathrm{T}$ stage, $\mathrm{M}$ stage, follicular helper $\mathrm{T}$ cells, Tregs, 


\begin{tabular}{|c|c|c|c|c|}
\hline Tumor-Infiltrating Immune Cell & Hazard Ratio & HR & $95 \%-\mathrm{CI}$ & P-value \\
\hline Mast cells resting & $\mapsto-1$ & 0.678 & {$[0.487 ; 0.943]$} & 0.021 \\
\hline Monocytes & $\mapsto$ & 0.701 & {$[0.503 ; 0.977]$} & 0.036 \\
\hline Dendritic cells resting & $\mapsto-1$ & 0.720 & {$[0.517 ; 1.003]$} & 0.052 \\
\hline Macrophages M2 & $\mapsto-1$ & 0.731 & {$[0.525 ; 1.017]$} & 0.063 \\
\hline Mast cells activated & $\longmapsto i$ & 0.746 & {$[0.415 ; 1.343]$} & 0.329 \\
\hline T cells CD4 memory resting & $\mapsto+1$ & 0.800 & {$[0.575 ; 1.114]$} & 0.187 \\
\hline Eosinophils & $\longmapsto$ & 0.893 & {$[0.497 ; 1.607]$} & 0.706 \\
\hline Dendritic cells activated & $\longmapsto$ & 0.934 & {$[0.568 ; 1.535]$} & 0.787 \\
\hline NK cells resting & $\longmapsto$ & 0.928 & {$[0.641 ; 1.344]$} & 0.694 \\
\hline $\mathrm{T}$ cells $\mathrm{CD8}$ & $\longmapsto$ & 0.969 & {$[0.696 ; 1.350]$} & 0.854 \\
\hline NK cells activated & $\mapsto$ & 1.008 & {$[0.724 ; 1.404 \mid$} & 0.962 \\
\hline$T$ cells CD4 memory activated & $\longrightarrow$ & 1.041 & {$[0.741 ; 1.464]$} & 0.816 \\
\hline Neutrophils & $\longmapsto$ & 1.050 & {$[0.753 ; 1.465]$} & 0.774 \\
\hline$B$ cells naive & $\longmapsto$ & 1.058 & $|0.759 ; 1.474|$ & 0.739 \\
\hline$T$ cells gamma delta & $=$ & 1.101 & {$[0.791 ; 1.534 \mid$} & 0.325 \\
\hline Plasma cells & $H=-1$ & 1.173 & {$[0.841 ; 1.636]$} & 0.883 \\
\hline Macrophages M1 & $r=-1$ & 1.186 & {$[0.852 ; 1.651]$} & 0.312 \\
\hline Macrophages M0 & $\stackrel{H}{H} \longrightarrow$ & 1.234 & {$[0.887 ; 1.718]$} & 0.212 \\
\hline B cells memory & $\Longrightarrow$ & 1.300 & {$[0.557 ; 3.000]$} & 0.544 \\
\hline$T$ cells follicular helper & $\longmapsto$ & 1.516 & {$[1.089 ; 2.111]$} & 0.014 \\
\hline $\mathrm{T}$ cells regulatory (Tregs) & $\longmapsto$ & 1.596 & [1.147; 2.222] & 0.006 \\
\hline
\end{tabular}
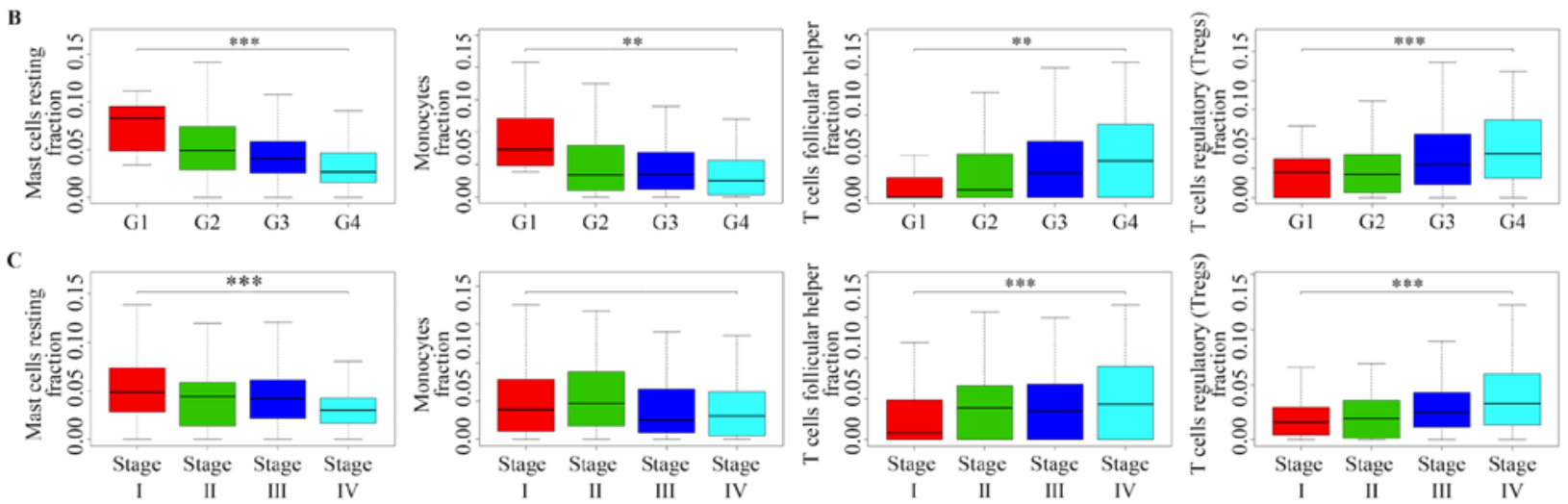

Figure 3. Prognostic value of TIICs in RCC. (A) Log-rank test for the prognostic associations of TIICs in RCC. (B) Association between four selected immune cell types and Fuhrman grade. ${ }^{* *} \mathrm{P}<0.01$ and ${ }^{* * * *} \mathrm{P}<0.001$. (C) Association between four selected immune cell types and the Tumor-Node-Metastasis classification. ${ }^{* * * *} \mathrm{P}<0.001$. CI, confidence interval; HR, hazard ratio; TIICs, tumor-infiltrating immune cells; RCC, renal cell carcinoma.

monocytes and resting mast cells were significant risk factors for RCC outcome (Fig. 4A). These factors were then incorporated into a multivariate analysis, and the results demonstrated that age $\geq 61$ years $(\mathrm{HR}=2.148 ; 95 \% \mathrm{CI}, 1.507-3.060 ; \mathrm{P}<0.001)$, M1 stage $(\mathrm{HR}=2.666 ; 95 \% \mathrm{CI}, 1.674-4.248 ; \mathrm{P}<0.001)$, a high proportion of Tregs (HR=1.437; 95\% CI, 1.008-2.049; $\mathrm{P}=0.045)$ and a low proportion of monocytes $(\mathrm{HR}=0.622 ; 95 \%$ CI, 0.435-0.890; $\mathrm{P}=0.009$ ) were separately associated with an increased risk of mortality (Fig. 4B).

Tregs infiltration in RCC samples. To further confirm the aforementioned results acquired for Tregs, the expression of the transcription factor FOXP3, which is the characteristic marker of Tregs, was assessed by IHC in 20 human RCC tissues and 20 paired adjacent tissues. As presented in Fig. 5, Treg density was significantly higher in RCC tissues compared with that in adjacent tissues, which was similar to the results from CIBERSORT analysis.

\section{Discussion}

Tumors are no longer considered as bulks of malignant cancer cells, but as a complex tumor microenvironments where other cell subpopulations corrupted by malignant neoplastic cells are recruited in order to constitute a self-sufficient biological structure (22). The tumor-related microenvironment comprises various cell populations, including immune cells, cancer-associated fibroblasts and endothelial cells (23). In addition, numerous chemokines, cytokines, proangiogenic mediators, growth factors and proteins of the extracellular matrix are involved in the interaction between these subpopulations of cells, such as immune cells, cancer-associated fibroblasts and endothelial cells, and cancer cells (24). The predominant cells recruited to and activated in the tumor microenvironment are immune cells (24). The immune system has been reported to possess cancer-inhibiting and cancer-promoting roles in the tumor microenvironment, and is associated with the process of cancer development (25-27). In RCC, a high variety of infiltrating immune cells has been reported. These immune cells can activate the immune response and are associated with clinical outcome. For example, a high level of CD8 ${ }^{+}$ $\mathrm{T}$ lymphocytes is associated with prolonged OS in patients with RCC (28).

In the present study, the results from the deconvolution of bulk gene expression data from TCGA cohort allowed the comprehensive analysis of the different immune cell infiltrations in RCC samples. Great differences in the immune cell composition between RCC and normal tissues were observed. Since CD4 naïve T cells were not infiltrated in the majority of samples, correlation analysis and subsequent survival analysis could not be performed. Furthermore, the association between these immune-infiltrated cells and the clinical outcome of 


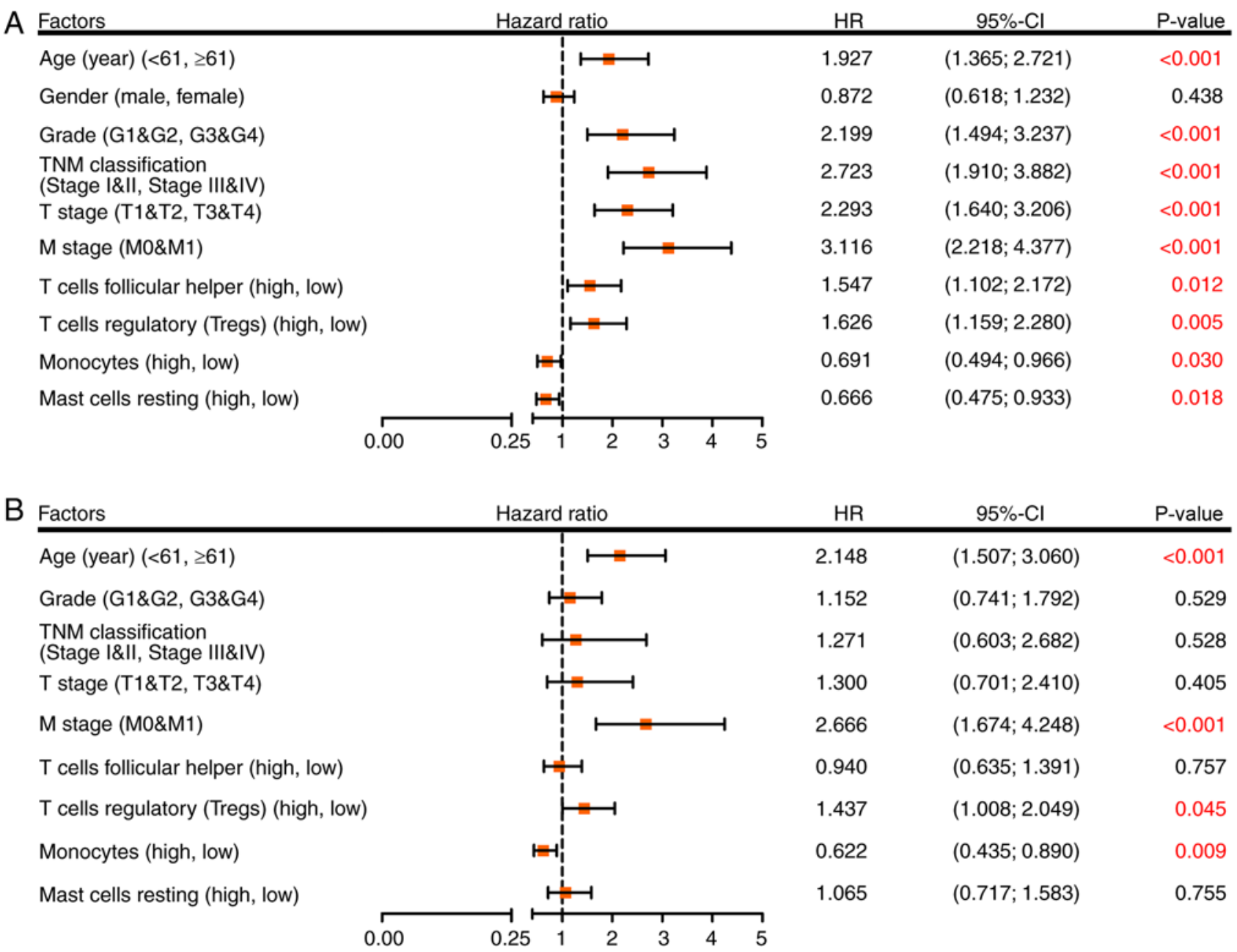

Figure 4. Cox regression model for overall survival in patients with RCC. (A) Association between four selected immune cell types and clinical information of patients with RCC analyzed via univariate Cox regression analysis. (B) Multivariate Cox regression analysis used to evaluate the independent risk factor of prognosis in patients with RCC. CI, confidence interval; HR, hazard ratio; RCC, renal cell carcinoma.
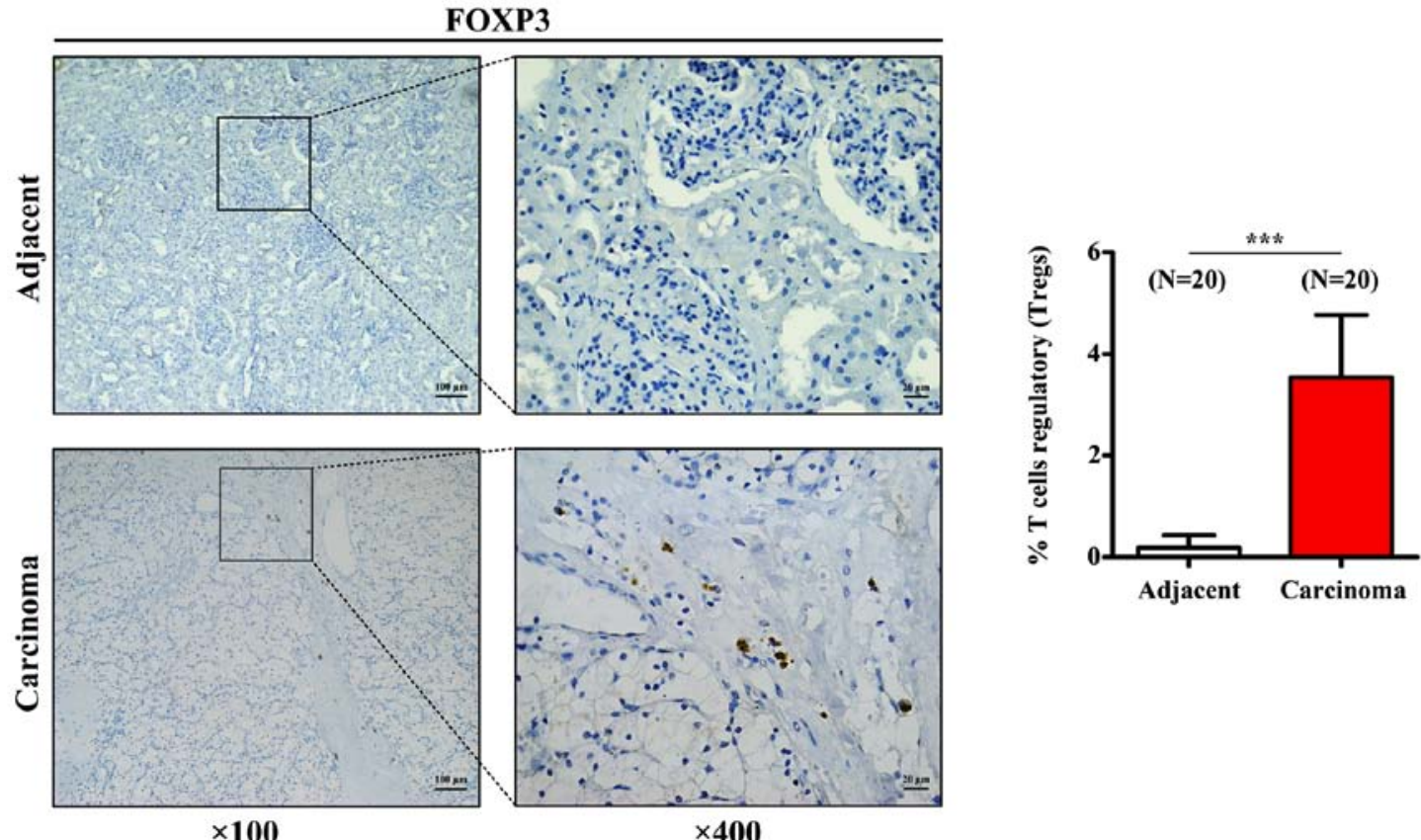

Figure 5. Representative images of FOXP3 immunohistochemical staining in RCC $(n=20)$ and paired adjacent tissues $(n=20)$. Histogram represents the density of regulatory T cells in RCC and adjacent tissues. Data are presented as the mean \pm standard deviation. ${ }^{* * *} \mathrm{P}<0.001$ vs. adjacent normal tissue. FOXP3, forkhead box P3; RCC, renal cell carcinoma. 
patients with RCC was investigated. The results demonstrated that a greater proportion of resting mast cells and monocytes was associated with a favorable prognosis, whereas a high level of follicular helper $\mathrm{T}$ cells and Tregs was associated with poor prognosis. In addition, following multivariate Cox regression analysis, Tregs and monocytes were determined as independent risk factors for the outcome of patients with RCC.

Tregs are adaptive immune cells that can suppress an excessive immune response and maintain self-tolerance and homeostasis (29). In cancer, Tregs can suppress the antitumor response, leading to tumor immune escape (30). A previous meta-analysis demonstrated that a high level of infiltration of FOXP $3^{+}$Tregs in the majority of solid tumors is significantly associated with poor prognosis of patients with cancer, including renal cancer (31). In patients with RCC, it has been reported that an increased number of Tregs in peripheral blood and tumors is negatively correlated with OS $(32,33)$. A high proportion of infiltrated Tregs in RCC may therefore indicate a worse outcome, which is similar to the CIBERSORT findings from the present study. Furthermore, the present study demonstrated that the proportion of Tregs was associated with tumor grade and TNM classification, which was also the case for other cancer types $(34,35)$. In addition, the results from IHC demonstrated that FOXP3 expression in Tregs was increased in RCC samples compared with that in adjacent tumors. Taken together, these findings suggested that Tregs may represent a high-risk factor for worse outcome in patients with RCC, and that decreasing Treg infiltration may be considered as a potential therapy in RCC.

Monocytes circulate in peripheral blood, migrate into tumor tissue and differentiate into different macrophage types in the tumor microenvironment (36). In humans, monocytes are heterogeneous and divided into three subsets, including classical, intermediate and non-classical, according to their expression of CD14 and CD16 (37). It is commonly considered that classical monocytes essentially differentiate into M1 macrophages, which contribute to the promotion of inflammation and tumor suppression (38). Conversely, intermediate and non-classical monocytes usually differentiate into M2 macrophages, which serve immunosuppressive and tumor-promoting roles (39). Furthermore, it has been demonstrated that the composition of the monocyte subset is altered in numerous diseases, including cancer $(40,41)$. Notably, the mutual conversion of M1 and M2 macrophage are valuable for prognosis (42). In the present study, monocytes were associated with positive prognosis in patients with RCC. However, several intersections in the survival curve of monocytes were highlighted, and the survival rate of the patients with RCC exhibiting a high monocyte count and those exhibiting a low monocyte count was reversed after $\sim 8$ years. These results may be due to changes in the composition of monocyte subsets, which may affect the prognosis of patients; however, this hypothesis requires further investigation.

In conclusion, the present study demonstrated that deconvolution of whole-tissue gene expression data by CIBERSORT provided refined information on the immune cell landscape of RCC samples. Furthermore, the different immunoprofiles between RCC and normal tissues may represent crucial tools that could be considered as potential targets for immunotherapies. In addition, the present study highlighted that TIICs may have an independent prognostic value in RCC. Coupling reliable deconvolution algorithms with large-scale genomic data, including TCGA cohort, may have the potential to reveal the biological and clinical implications of any cell that infiltrates the tumor microenvironment in RCC.

\section{Acknowledgements}

Not applicable.

\section{Funding}

The present study was supported by the National Natural Science Foundation of China (grant. no. 81874092).

\section{Availability of data and materials}

All data generated or analyzed during this study are included in this published article.

\section{Authors' contributions}

XG and WH designed the study. GZ and XG prepared the figures and drafted the manuscript. GZ, LP, HY, FL, XL and $\mathrm{XZ}$ performed the experiments and analyzed the data. XG and WH provided reagents and analytic tools, and revised the manuscript. All authors have read and approved the final version of the manuscript.

\section{Ethics approval and consent to participate}

The present study was approved by the Ethics Committee of the First Affiliated Hospital of Chongqing Medical University, and all patients provided written informed consent.

\section{Patient consent for publication}

Not applicable.

\section{Competing interests}

The authors declare that they have no competing interests.

\section{References}

1. Siegel RL, Miller KD and Jemal A: Cancer statistics, 2018. CA Cancer J Clin 68: 7-30, 2018.

2. Capitanio U, Bensalah K, Bex A, Boorjian SA, Bray F, Coleman J, Gore JL, Sun M, Wood C and Russo P: Epidemiology of renal cell carcinoma. Eur Urol 75: 74-84, 2019.

3. Fisher R, Gore M and Larkin J: Current and future systemic treatments for renal cell carcinoma. Semin Cancer Biol 23: 38-45, 2013.

4. Climent MA, Munoz-Langa J, Basterretxea-Badiola L and Santander-Lobera C: Systematic review and survival meta-analysis of real world evidence on first-line pazopanib for metastatic renal cell carcinoma. Crit Rev Oncol Hematol 121: 45-50, 2018.

5. Candido J and Hagemann T: Cancer-related inflammation. J Clin Immunol 33 (Suppl 1): S79-S84, 2013.

6. Swann JB and Smyth MJ: Immune surveillance of tumors. J Clin Invest 117: 1137-1146, 2007.

7. Thompson RH, Dong H, Lohse CM, Leibovich BC, Blute ML, Cheville JC and Kwon ED: PD-1 is expressed by tumor-infiltrating immune cells and is associated with poor outcome for patients with renal cell carcinoma. Clin Cancer Res 13: 1757-1761, 2007. 
8. Yoshihara K, Shahmoradgoli M, Martinez E, Vegesna R, Kim H, Torres-Garcia W, Treviño V, Shen H, Laird PW, Levine DA, et al: Inferring tumour purity and stromal and immune cell admixture from expression data. Nat Commun 4: 2612, 2013.

9. Vogelzang NJ, Priest ER and Borden L: Spontaneous regression of histologically proved pulmonary metastases from renal cell carcinoma: A case with 5-year followup. J Urol 148: 1247-1248, 1992.

10. Janiszewska AD, Poletajew S and Wasiutynski A: Spontaneous regression of renal cell carcinoma. Contemp Oncol (Pozn) 17: 123-127, 2013.

11. Considine B and Hurwitz ME: Current status and future directions of immunotherapy in renal cell carcinoma. Curr Oncol Rep 21: 34, 2019.

12. Escudier B: Emerging immunotherapies for renal cell carcinoma. Ann Oncol 23 (Suppl 8): viii35-viii40, 2012.

13. Amin A, Dudek AZ, Logan TF, Lance RS, Holzbeierlein JM, Knox JJ, Master VA, Pal SK, Miller WH Jr, Karsh LI, et al: Survival with AGS-003, an autologous dendritic cell-based immunotherapy, in combination with sunitinib in unfavorable risk patients with advanced renal cell carcinoma (RCC): Phase 2 study results. J Immunother Cancer 3: 14, 2015.

14. Bingle L, Brown NJ and Lewis CE: The role of tumour-associated macrophages in tumour progression: Implications for new anticancer therapies. J Pathol 196: 254-265, 2002.

15. Fridman WH, Pages F, Sautes-Fridman C and Galon J: The immune contexture in human tumours: Impact on clinical outcome. Nat Rev Cancer 12: 298-306, 2012.

16. Schraml P, Athelogou M, Hermanns T, Huss R and Moch $\mathrm{H}$ : Specific immune cell and lymphatic vessel signatures identified by image analysis in renal cancer. Mod Pathol 32: 1042-1052, 2019.

17. Liss MA, Chen Y, Rodriguez R, Pruthi D, Johnson-Pais T, Wang H, Mansour A and Kaushik D: Immunogenic heterogeneity of renal cell carcinoma with venous tumor thrombus. Urology 124: 168-173, 2019.

18. Newman AM, Liu CL, Green MR, Gentles AJ, Feng W, Xu Y, Hoang CD, Diehn $M$ and Alizadeh AA: Robust enumeration of cell subsets from tissue expression profiles. Nat Methods 12: 453-457, 2015

19. Rohr-Udilova N, Klinglmuller F, Schulte-Hermann R, Stift J, Herac M, Salzmann M, Finotello F, Timelthaler G, Oberhuber G, Pinter M, et al: Deviations of the immune cell landscape between healthy liver and hepatocellular carcinoma. Sci Rep 8: 6220, 2018.

20. Wei L, Jin Z, Yang S, Xu Y, Zhu Y and Ji Y: TCGA-assembler 2: Software pipeline for retrieval and processing of TCGA/CPTAC data. Bioinformatics 34: 1615-1617, 2018.

21. Smyth GK: Limma: Linear models for microarray data In: Bioinformatics and Computational Biology Solutions Using R and Bioconductor. Gentleman R, Carey VJ, Huber W, Irizarry RA and Dudoit S (eds). Statistics for Biology and Health Springer New York, New York, NY, pp397-420, 2005.

22. Peltanova B, Raudenska M and Masarik M: Effect of tumor microenvironment on pathogenesis of the head and neck squamous cell carcinoma: A systematic review. Mol Cancer 18: $63,2019$.

23. Senbabaoglu Y, Gejman RS, Winer AG, Liu M, Van Allen EM, de Velasco G, Miao D, Ostrovnaya I, Drill E, Luna A, et al: Tumor immune microenvironment characterization in clear cell renal cell carcinoma identifies prognostic and immunotherapeutically relevant messenger RNA signatures. Genome Biol 17: 231, 2016

24. Bremnes RM, Al-Shibli K, Donnem T, Sirera R, Al-Saad S, Andersen S, Stenvold H, Camps C and Busund LT: The role of tumor-infiltrating immune cells and chronic inflammation at the tumor site on cancer development, progression, and prognosis: Emphasis on non-small cell lung cancer. J Thorac Oncol 6: 824-833, 2011.

25. Ishigami S, Natsugoe $S$, Tokuda K, Nakajo A, Che $X$, Iwashige $H$, Aridome K, Hokita S and Aikou T: Prognostic value of intratumoral natural killer cells in gastric carcinoma. Cancer 88: $577-583,2000$

26. Ribatti D, Ennas MG, Vacca A, Ferreli F, Nico B, Orru S and Sirigu P: Tumor vascularity and tryptase-positive mast cells correlate with a poor prognosis in melanoma. Eur J Clin Invest 33: 420-425, 2003.
27. de Visser KE, Eichten A and Coussens LM: Paradoxical roles of the immune system during cancer development. Nat Rev Cancer 6: 24-37, 2006

28. Yao J, Xi W, Zhu Y, Wang H, Hu X and Guo J: Checkpoint molecule PD-1-assisted CD8 ${ }^{+} \mathrm{T}$ lymphocyte count in tumor microenvironment predicts overall survival of patients with metastatic renal cell carcinoma treated with tyrosine kinase inhibitors. Cancer Manag Res 10: 3419-3431, 2018.

29. Lahl K, Loddenkemper C, Drouin C, Freyer J, Arnason J, Eberl G, Hamann A, Wagner H, Huehn J and Sparwasser T: Selective depletion of Foxp3 ${ }^{+}$regulatory $\mathrm{T}$ cells induces a scurfy-like disease. J Exp Med 204: 57-63, 2007.

30. Sakaguchi S, Miyara M, Costantino CM and Hafler DA: FOXP3 ${ }^{+}$ regulatory $\mathrm{T}$ cells in the human immune system. Nat Rev Immunol 10: 490-500, 2010.

31. Shang B, Liu Y, Jiang SJ and Liu Y: Prognostic value of tumor-infiltrating FoxP ${ }^{+}$regulatory $\mathrm{T}$ cells in cancers: A systematic review and meta-analysis. Sci Rep 5: 15179, 2015.

32. Griffiths RW, Elkord E, Gilham DE, Ramani V, Clarke N, Stern PL and Hawkins RE: Frequency of regulatory T cells in renal cell carcinoma patients and investigation of correlation with survival. Cancer Immunol Immunother 56: 1743-1753, 2007.

33. Finke JH, Rini B, Ireland J, Rayman P, Richmond A, Golshayan A, Wood L, Elson P, Garcia J, Dreicer R and Bukowski R: Sunitinib reverses type-1 immune suppression and decreases T-regulatory cells in renal cell carcinoma patients. Clin Cancer Res 14: 6674-6682, 2008.

34. Papaioannou E, Sakellakis M, Melachrinou M, Tzoracoleftherakis E, Kalofonos H and Kourea E: A Standardized Evaluation Method for FOXP3 ${ }^{+}$Tregs and $\mathrm{CD} 8^{+} \mathrm{T}$-cells in breast carcinoma: Association with breast carcinoma subtypes, stage and prognosis. Anticancer Res 39: 1217-1232, 2019.

35. Seminerio I, Descamps G, Dupont S, de Marrez L, Laigle JA, Lechien JR, Kindt N, Journe F and Saussez S: Infiltration of FoxP $3^{+}$regulatory $\mathrm{T}$ cells is a strong and independent prognostic factor in head and neck squamous cell carcinoma. Cancers (Basel) 11: pii: E227, 2019.

36. van Furth R and Cohn ZA: The origin and kinetics of mononuclear phagocytes. J Exp Med 128: 415-435, 1968.

37. Ziegler-Heitbrock L, Ancuta P, Crowe S, Dalod M, Grau V, Hart DN, Leenen PJ, Liu YJ, MacPherson G, Randolph GJ, et al: Nomenclature of monocytes and dendritic cells in blood. Blood 116: e74-e80, 2010

38. Gui T, Shimokado A, Sun Y, Akasaka T and Muragaki Y: Diverse roles of macrophages in atherosclerosis: From inflammatory biology to biomarker discovery. Mediators Inflamm 2012: 693083, 2012

39. Lee HW, Choi HJ, Ha SJ, Lee KT and Kwon YG: Recruitment of monocytes/macrophages in different tumor microenvironments. Biochim Biophys Acta 1835: 170-179, 2013.

40. Movahedi K, Laoui D, Gysemans C, Baeten M, Stangé G, Van den Bossche J, Mack M, Pipeleers D, In't Veld P, De Baetselier P and Van Ginderachter JA: Different tumor microenvironments contain functionally distinct subsets of macrophages derived from Ly6C(high) monocytes. Cancer Res 70: 5728-5739, 2010.

41. Mantovani A, Sozzani S, Locati M, Allavena P and Sica A: Macrophage polarization: Tumor-associated macrophages as a paradigm for polarized M2 mononuclear phagocytes. Trends Immunol 23: 549-555, 2002.

42. Romano E, Kusio-Kobialka M, Foukas PG, Baumgaertner P, Meyer C, Ballabeni P, Michielin O, Weide B, Romero P and Speiser DE: Ipilimumab-dependent cell-mediated cytotoxicity of regulatory $\mathrm{T}$ cells ex vivo by nonclassical monocytes in melanoma patients. Proc Natl Acad Sci USA 112: 6140-6145, 2015.

This work is licensed under a Creative Commons Attribution-NonCommercial-NoDerivatives 4.0 International (CC BY-NC-ND 4.0) License. 